Objectives To report updated results on long-term nightshift work and breast cancer risk in Hong Kong women.

Method This ongoing case-control study involves three hospitals in Hong Kong. By 31/03/2013, we've consecutively recruited 443 newly diagnosed breast cancer cases and 335 age-matched controls from the hospital that the cases came from, with a response rate of $90 \%$. We expect to collect 1000 cases and 1000 controls by $31 / 12 / 2013$. We obtained each participant's lifetime occupational history and shift work, exposure to light-at-night and other important risk factors including family cancer history. We performed unconditional logistic regression analyses to calculate odds ratio (OR) after adjusting for potential confounders.

Results The age at diagnosis (interview) between cases and controls is comparable $(55.1 \pm 11.9$ vs. $54.2 \pm 14.6$ years $)$. More cases than controls were non-parity and non-breast feeding, but gave first birth slightly late. A significantly elevated (adjusted $\mathrm{OR}=1.90,95 \% \mathrm{CI}: 1.24-2.89$ ) breast cancer risk was observed in never employed women. Among those ever employed, 19.8\% of breast cancers had ever worked at nightshift at least once per month for $\geq 1$ year and it was $21.7 \%$ for the controls. Further analyses revealed that nightshift work for $\geq 15$ years resulted in an adjusted OR of 1.55 (95\% CI: $0.76-3.14)$ but power is limited. There is no excess breast cancer risk for women with nightshift work for $<15$ years.

Conclusions This ongoing study provides supportive evidence on a positive association between long-term nightshift work and breast cancer risk. [Research Grants Council (Project no.: 474811) and Direct Grant (Project no.: 2041788), shelly@cuhk. edu.hk].

\section{EXAMINING MULTIPLE EXPOSURE PATHWAYS OF BERYLLIUM USING MIXED AND STRUCTURAL EQUATION MODING TECHNIQUES}

'Jenna Armstrong, ${ }^{1}$ Abbas Virji, ${ }^{2}$ Mary Davis, 'Gregory Day, 'Aleksandr Stefaniak, 1 Marcia Stanton, ${ }^{3}$ David Deubner, ${ }^{1}$ Christine Schuler. ${ }^{1}$ National Institute of Occupational Safety and Health, Morgantown, WV, USA; '2Tufts University, Boston, MA, USA; ${ }^{3}$ Materion Brush, Inc., Elmore, OH, USA

10.1136/oemed-2014-102362.24

Objectives Inhalation beryllium exposures are associated with sensitisation, however dermal exposures are also important. In a previous study, we identified strong correlations between dermal-air, dermal- surface, and air- surface measurements. The aim of this study was to investigate workplace factors associated with exposures using mixed-effects models and structural equation modelling (SEM).

Method Beryllium was measured in personal air, on gloves, and on surfaces at three manufacturing facilities. Predictor variables included substance and activity emission potential (REACH classification), dilution, segregation, PPE, personal behaviour, and work shift.

Results The mixed model described 57 and 59\% of total variance for air and dermal, respectively. The total variance explained by the SEM model for air and dermal was 0.51 and $0.48 \%$ respectively. In both models activity and substance emission potential, surface contamination, dilution, and personal behaviour were significant predictors of air concentrations $(\mathrm{p} \leq$ 0.05 ); and surface contamination and air concentrations were significant predictors of dermal loading on cotton gloves $(\mathrm{p} \leq$ $0.05)$. However, work shift and personal behaviour were predictive of dermal loading in the SEM ( $\mathrm{p} \leq 0.03)$, but not in the mixed model. In addition, the SEM reported a parameter estimate for air concentration as a predictor of dermal loading that was an order of magnitude higher than in the mixed model. Conclusions Although SEM requires relatively large sample sizes, it is useful for modelling multiple, correlated dependent variables. In addition, full-information maximum likelihood (FIML) methods can be used in SEM to include missing predictor variable data. Although we found both models to be useful, SEM has the potential to illustrate indirect pathways of outcome variables.

\section{PREGNANCY OUTCOMES FOR WOMEN EMPLOYED AS HAIRDRESSERS, COSMETOLOGISTS AND LABORATORY WORKERS-SYSTEMATIC REVIEW OF THE LITERATURE AND DATA- ANALYSIS OF FINNISH MATERNITY BIRTH REGISTRY}

Jacqueline Halliday-Bell. Institute of Occupational and Environmental Medicine, Birmingham, UK

\subsection{6/oemed-2014-102362.25}

Objectives This study examines pregnancy outcomes in Finland: gender differentiation, low birth weight, high birth weight, preterm delivery and post-term delivery, small-for-gestational-age, large-for-gestational-age, stillbirth and early neonatal death for study occupations compared to controls

Method Among 507659 prima gravida women were 12854 hairdressers, 1841 cosmetologists and 3587 laboratory workers. The control populations consisted of 40405 teachers, 1968 musicians and 447004 women from the general population.

Adjusted odds ratios (aOR) were calculated using binomial logistic regression analysis. Adjustments made: maternal age, smoking habit, marital status, socioeconomic status.

Results aOR for male births was lower for hairdressers than teachers 0.92 (95\% CI $0.87-0.98)$. aOR for post-term delivery was greater for cosmetologists compared to teachers, $2.13(95 \%$ CI 1.04-4.35).

Crude odds ratios (ORs) were also statisitically significant Cosmetologists compared to musicians for ratio of male/female births, OR 1.04 (95\% CI 1.00-1.08). Haidressers compared to the general public: small for gestational age, OR 1.01 (95\% CI 1.00-1.02), large for gestational age, OR 1.02 (95\% CI 1.001.03 ) and post term delivery OR 1.06 (95\% CI 1.02-1.11).

The statisitically significant results for pooled effect size (ES) from the meta-analysis: low birth weight in hairdressers, ES 1.1 (95\% CI 1.01-1.20) and small-for-gestational-age infants among hairdressers ES 1.18 (95\% CI,1.08-1.30).

The pooled effect size for large for gestational age infants among hairdressers was statisitically significantly reduced for LGA infants among hairdressers, ES 0.71 (95\% CI 0.55-0.91).

Conclusions Hairdressers, increased risk of certain pregnancy outcomes. Policy recommendations to improve protection forpregnant workers have been made.

\section{NERVOUS SYSTEM DISEASE AMONG KOREAN WORKERS EXPOSED TO MANGANESE}

Yeon-Soon Ahn. Dongguk University Ilsan Hospital, Goyang, Republic of Korea

\subsection{6/oemed-2014-102362.26}

Objectives Exposure to excess levels of manganese in occupational settings has been associated with a neurological syndrome. So morbidity of nervous system disease among Korean workers exposed to manganese described through this large cohort study. 
Method Using medical surveillance data, hospital admission of nervous system disease (GOO-G99) from 2000 to 2005 was analysed in cohort contained manganese exposed male workers (438,693 person years). Also, 2\% of Korean men was randomly selected and analysed their hospital admission data. Standardised Admission Ratios (SAR) of nervous disease among manganese exposed workers was estimated reference to Korean men.

Results For 6 years, 500 admissions with nervous system diseases (G00-G99) were observed in solvents exposed workers. SARs for overall nervous diseases (G00-G09) (SAR $=1.24$, 95\% CI 1.131.35), inflammatory disease of CNS (G00-G09) (SAR $=1.92,95 \%$ CI 1.52-2.39), other degenerative diseases of nervous system (G31) (SAR $=3.60,95 \%$ CI 1.16-8.40) and nerve, nerve root and plexus disorders (G50-G59) (SAR $=1.66,95 \%$ CI 1.36-2.00) were significantly higher than those of Korean men. SAR of extrapyramidal and movement disorders (G20-G26) was significantly high $(\mathrm{SAR}=2.03,95 \% \mathrm{CI}=1.05-3.55)$ among workers with 10 and more years employment duration.

Conclusions This manganese exposed workers' cohort with short follow-up periods exhibits significantly elevated admission with overall and some kinds of nervous disease comparing to Korean men. Especially, increased SAR of extrapyramidal and movement disorder suggests relatedness of manganese exposure.

\section{MAGNETIC FIELD EXPOSURES AND BRAIN TUMOUR AND LEUKAEMIA RISKS IN UK ELECTRICITY GENERATION AND TRANSMISSION WORKERS, 1973- 2010}

Tom Sorahan. University of Birmingham, Birmingham, UK

10.1136/oemed-2014-102362.27

Objectives To investigate whether brain tumour or leukaemia risks are related to occupational exposure to low-frequency magnetic fields.

Method Brain tumour and leukaemia risks experienced by 73 051 UK electricity supply industry workers were investigated for the period 1973-2010. All employees were hired in the period 1952-1982 and were employed for at least six months with some employment in the period 1973-1982. Detailed calculations had been performed to assess exposures to magnetic fields. Poisson regression was used to calculate relative risks (rate ratios) of developing a brain tumour (or glioma or meningioma) or leukaemia (or its sub-types) for categories of lifetime, distant (lagged) and recent (lugged) exposure.

Results Findings for gliomas, all brain tumours combined, and all leukaemia were unexceptional; risks were close to (or below) unity for all exposure categories. There were no significant doseresponse effects shown for meningioma, but there was some evidence of elevated risks in the three highest exposure categories for distant exposures. There were no significant dose-response effects shown for the main leukaemia sub-types, but there was a significant positive trend for acute lymphocytic leukaemia (ALL). National comparisons indicated that the limited associations shown for meningioma and ALL were based, in the main, on unusually low risks in the lowest exposure category.

Conclusions The findings are consistent with the hypotheses that both distant and recent magnetic field exposures are not causally related to gliomas or to the main leukaemia sub-types. The limited positive findings for meningioma and ALL may be chance findings; national comparisons argue against a causal interpretation.
0091 INFLAMMATORY MARKERS AND EXPOSURE TO AIR POLLUTANTS AMONG WORKERS IN A SWEDISH PULP AND PAPER MILL

${ }^{1}$ Håkan Westberg, ${ }^{2}$ Karine Elihn, ${ }^{3}$ Eva Andersson, ${ }^{4}$ Bodil Persson, ${ }^{5}$ Ing-Liss Bryngelsson, ${ }^{6}$ Bengt Sjögren. ${ }^{1}$ Department of Occupational and Environmental Medicine, Örebro University, Örebro, Sweden; ${ }^{2}$ Department of Applied Environmental Science, Stockhlm University, Stockholm, Sweden; ${ }^{3}$ Department of Occupational and Environmental Medicine, Sahlgrenska University, Gothenburg, Sweden; ${ }^{4}$ Department of Occupational and Environmental Medicine Lund University, Lund, Sweden; ${ }^{5}$ Department of Occupational and Environmental Medicine, Örebro University Hospital, Örebro, Sweden; ${ }^{6}$ Work Environment Toxicology, Institute of Environmental Medicine, Karolinska Institute, Stockholm, Sweden

\subsection{6/oemed-2014-102362.28}

Objectives Study the relationship between inhalation of airborne particles in a pulp and paper mill and markers of inflammation and coagulation in blood.

Method Personal sampling of inhalable dust was performed for 72 subjects working in a Swedish pulp and paper mill. Stationary measurements were used to study different particle size fractions including respirable dust, $\mathrm{PM}_{10}, \mathrm{PM}_{2.5}$, the particle surface area and particle number concentrations of ultrafine particles. Markers of inflammation such as interleukins (IL-1b, IL-6, IL-8, and IL-10), C-reactive protein (CRP), serum amyloid A (SAA), and fibrinogen and markers of coagulation such as factor VIII, von Willebrand factor $\mathrm{vWF}$ ), plasminogen activator inhibitor (PAI-1), and D-dimer were determined before the first shift after a work free period of normally five days and after the first, second and third shift

Results The average $8 \mathrm{hr}$-TWA level of inhalable dust in was $0.30 \mathrm{mg} / \mathrm{m}^{3}$, range $0.005-3.3 \mathrm{mg} / \mathrm{m}^{3}$. The proxies for $8 \mathrm{hr}-\mathrm{TWAs}$ of respirable dust was $0.045 \mathrm{mg} / \mathrm{m}^{3}, \mathrm{PM}_{10} 0.17 \mathrm{mg} / \mathrm{m}^{3}$ and $\mathrm{PM}_{2.5} 0.08 \mathrm{mg} / \mathrm{m}^{3}$. No significant increase of markers of inflammation or coagulation in blood during the working week was noted after a non-exposure period of five days. In a multiple regression analysis adjustments were made for sex, age, smoking, BMI, and blood group. Significant positive correlations were found between several particle exposure metrics and CRP, SAA and fibrinogen taken pre- and post-shift day 1, suggesting a dose-effect relationship.

Conclusions These relations between particle exposure and inflammatory markers may indicate an increased risk of cardiovascular disease.

\section{BLADDER CANCER INCIDENCE AMONG WORKERS EXPOSED TO O-TOLUIDINE, ANILINE AND NITROBENZENE AT A RUBBER CHEMICAL MANUFACTURING PLANT}

${ }^{1}$ Tania Carreón, ${ }^{1}$ Misty Hein, ${ }^{1}$ Kevin Hanley, ${ }^{2}$ Susan Viet, ${ }^{1}$ Avima Ruder. ${ }^{1}$ National Institute for Occupational Safety and Health, Cincinnati, Ohio, USA; ${ }^{2}$ Westat, Rockville, Maryland, USA

\subsection{6/oemed-2014-102362.29}

Objectives An earlier investigation found increased bladder cancer incidence among workers at a rubber chemical manufacturing plant that used o-toluidine, aniline and nitrobenzene. The cohort was expanded to include additional workers $(\mathrm{n}=1875)$ and updated through 2007 to assess bladder cancer with improved exposure characterisation.

Method Work histories were updated and exposure categories and ranks were developed for o-toluidine, aniline and nitrobenzene combined. Incident cancers were identified by linkage to six state cancer registries. Residency in time-dependent cancer 